\title{
Simulation Analysis of Fault Feature Extraction and Fusion for Analog Circuits Based on Information Fusion
}

\author{
Shi Bao and Jun Xu \\ The Academy of Armored Forces Engineering,Beijing,100072,China
}

\begin{abstract}
In the fault diagnosis of analog circuit, the fault feature extraction is a very important link, and the results of the extraction directly impact on the accuracy of the final fault diagnosis. Because of the limitation of single fault feature extraction method, We used wavelet packet analysis and principal component analysis (PCA) to extract fault features simultaneously in this paper, and constructed three different feature vector fusion models. The results of the fusion models are then fed into a fault classifier model based on support vector machines to obtain the diagnostic results. The simulation results show that the proposed method can effectively improve the correctness of fault diagnosis compared with single fault feature extraction method.
\end{abstract}

Keywords-simulation; extraction; fusion models; fault diagnosis

\section{INTRODUCTION}

In the research of analog circuit fault diagnosis, How to quickly and effectively extract the fault characteristics that reflect the state of the circuit is the difficulty and key for circuit fault diagnosis and testing. Whether the extracted fault feature can fully reflect all the important information of the original failure sample has a great impact on the diagnosis results. There are many kinds of fault feature extraction methods now, such as principal component analysis (PCA), wavelet analysis, feature extraction based on statistical theory, Empirical mode decomposition, local mean decomposition ${ }^{[1]}$. Each feature extraction method has its own unique advantages, but also has some limitations, which often lead to the loss of some of the details of the information or feature vector higher dimensions. Based on the idea of information fusion, we use principal component analysis (PCA) and wavelet packet analysis to extract the fault samples at the same time and fuse the feature vectors after that. This method can fully extract the features of fault samples. Among them, the principal component analysis method can effectively reduce the dimension under the premise of preserving the important information of the sample, which greatly reducing the calculation amount ${ }^{[2]}$. And Wavelet packet analysis is an extension of wavelet analysis, enabling a more precise and accurate analysis of the signal. After the extraction, the feature vectors extracted by the two methods are merged according to the model proposed in this paper, and the fusion feature vectors are fed into the support vector machine classifier for training and diagnosis. The advantage of this method is to use the fault sample information as much as possible in order to ensure the final diagnosis is more accurate.

\section{FAult FeAture EXTRACTION MEHOd FOR ANALOG CIRCUITS}

\section{A. Principal Component Analysis(pca)}

Assuming that the original data sample is $X=\left(x_{i j}\right)_{m \times n} \mathrm{~m}$ is the dimension of each sample data, $\mathrm{n}$ is the number of samples. The normalized data matrix $Y=\left(y_{i j}\right)$ is obtained by normalizing $\mathrm{X}$, among them;

$$
\begin{gathered}
y_{i j}=\frac{x_{i j}-\overline{x_{i}}}{\sqrt{S_{i}}} \quad \mathrm{i}=1,2, \cdots \mathrm{m} ; \mathrm{j}=1,2, \cdots \mathrm{n} \\
\overline{x_{i}}=\frac{1}{n} \sum_{j=1}^{n} x_{i j} \quad S_{i}=\frac{1}{n} \sum_{j=1}^{n}\left(x_{i j}-\overline{x_{i}}\right)^{2}
\end{gathered}
$$

And then the correlation matrix $R$ of $Y$ is obtained. Then the eigenvalues $\lambda_{i}$ of the matrix R and the eigenvectors $a_{i}$ of the corresponding eigenvalues are calculated by Matlab software. The $\lambda_{i}$ should be arranged from large to small. At last, Calculate the cumulative principal component contribution rate of the previous $r$ eigenvalues.

$$
\mathrm{R}=\frac{1}{n} Y Y^{T} \quad \mathrm{~T} R_{r}=\sum_{k=1}^{r} \lambda_{k} / \sum_{i=1}^{m} \lambda_{i}
$$

Finally, the eigenvectors corresponding to the first $r$ eigenvalues with cumulative variance contribution rate of more than $90 \%$ are selected to form the characteristic subspace.

\section{B. Wavelet Packet Analysis Method}

Wavelet packet analysis can express the frequency characteristics which is unconspicuous using the energy form 
that is obviously changes, then the feature vectors of each fault mode are constructed according to this energy[3]. Specific steps are as follows:

(1) The output signal $\mathrm{S}$ is decomposed by n-layer wavelet packet, and a total of $2^{n}$ wavelet packet decomposition coefficients $X_{i}$ are extracted.

(2) According to the wavelet packet coefficients, the each frequency band signal $\mathrm{Si}$ is reconstructed.

$$
S=\sum_{i=0}^{2 n-1} S_{i}
$$

(3) Calculate the total energy of each band signal. $E_{i}$ denotes the energy of $S_{i}$

$$
E_{i}=\int\left|S_{i}\right|^{2} d t=\sum_{k=1}^{N}\left|x_{i}(k)\right|^{2}
$$

$x_{i}(k)$ is the amplitude of the discrete point $S_{i}$, the number of signals in the frequency band (length) is $\mathrm{N}$.

(4) Constructs an feature vector $\mathrm{T}$ with energy as its element according to $E_{i}$ and normalizes it[4].

$$
\mathrm{T}=\left[E_{0}, E_{1}, \cdots E_{2 n-1}\right]
$$

After normalization

$$
\mathrm{t}=\left[E_{0}, E_{1}, \cdots E_{2 n-1}^{\vee}\right]
$$

Among them

$$
E_{i}=E_{i} / E \quad E=\left(\sum_{j=0}^{2 n-1}\left|E_{i}\right|^{2}\right)^{1 / 2}
$$

\section{Selection of Optimal Wavelet Bases}

The characteristic deviation degree $D_{b}$ represents the degree of deviation between the fault feature and the normal state feature. In this paper, will use this as the selection criteria to determine the optimal wavelet basis [5].

$$
D_{b}=\sum_{i=1}^{s} \sum_{j=1}^{N} P_{i}\left(m_{j}^{i}-m_{j}\right)\left(m_{j}^{i}-m_{j}\right)^{T}
$$

$\mathrm{Pi}$ denotes the prior probability of the i-th fault characteristic, $m_{j}^{i}$ represents the $\mathrm{j}$-th data in the i-th set of fault feature samples, mj represents the $\mathrm{j}$-th data of the normal state sample set.

\section{FEATURE EXTRACTION AND FUSION BASED ON INFORMATION FUSION}

The following figure is a block diagram of feature extraction and fusion:

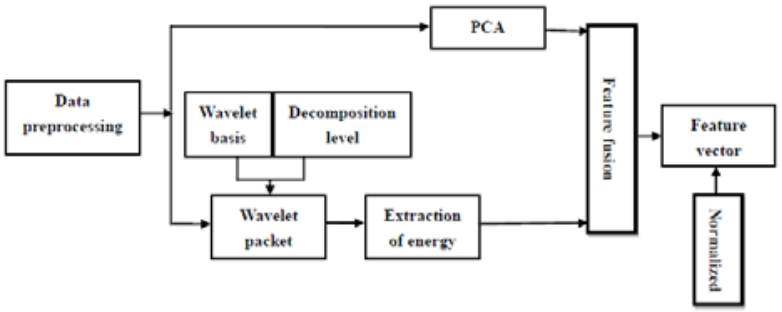

FIGURE I. BLOCK DIAGRAM OF FEATURE EXTRACTION AND FUSION

Specific feature extraction and fusion method steps are as follows:

(1) Multisim software was used to model the diagnosis circuit. Sensitivity analysis was used to select the components with high sensitivity for fault setting.

(2) Pre-processing the collected sample data, and make it do the difference operation with standard response signal, and a difference signal $X_{d}$ is obtained[6]. Supposing the original sample data is $\mathrm{X}=\left(x_{1}, x_{2}, \cdots, x_{n}\right)$, The sample data for the standard fault-free signal is $\left(x_{s}\right)_{m \times 1}$.

$$
X_{d}=\left(x_{1}-x_{s}, x_{2}-x_{s}, \cdots, x_{n}-x_{s}\right)
$$

(3) Do PCA feature extraction for Xd and to build a new feature vector.

(4) Do wavelet packet energy extraction for the difference signal $X_{d}$ and to build a new feature vector.

(5) The PCA feature and the wavelet energy feature are merged to form the feature vector, and normalized. In this paper, three different fusion models are proposed, and the effects of different fusion model factors $\mu$ on the results are also studied.

Assuming that the number of principal elements selected by PCA is $r$, it forms an r-dimensional feature vector $\mathrm{a}=\left[a_{1}, a_{2}, \cdots a_{r}\right]^{T}$. The wavelet packet decomposition layer is $\mathrm{n}$, and the final extracted energy feature vector is $\mathrm{t}$ dimension which is $b=\left[b_{1}, b_{2}, \cdots b_{t}\right]^{T}$.In the fusion models, $0<\mu<1, \beta=1-\mu$.The fused feature vector is $\mathrm{c}$, 
(5) The overall-cross model (W):

$$
\mathrm{c}=[\mu a, \beta b]^{T}=\left[\begin{array}{l}
\mu a \\
\beta b
\end{array}\right]
$$

(6) The one -one cross model (O):

$$
c=\left\{\begin{array}{l}
{\left[\mu a_{1}, \beta b_{1}, \mu a_{2}, \beta b_{2}, \cdots, \mu a_{r}, \beta b_{t}\right]^{T}(r=t)} \\
{\left[\mu a_{1}, \beta b_{1}, \mu a_{2}, \beta b_{2}, \cdots, \mu a_{t}, \beta b_{t}, \mu a_{t+1}, \cdots, \mu a_{r}\right]^{T}(r>t)} \\
{\left[\mu a_{1}, \beta b_{1}, \mu a_{2}, \beta b_{2}, \cdots, \mu a_{r}, \beta b_{r}, \beta b_{r+1}, \cdots, \beta b_{t}\right]^{T}(r<t)}
\end{array}\right.
$$

(3) The two-two cross model (T):

$$
c=\left\{\begin{array}{l}
{\left[\mu a_{1}, \mu a_{2}, \beta b_{1}, \beta b_{2}, \cdots, \beta b_{t}\right]^{T}(r=t)} \\
{\left[\mu a_{1}, \mu a_{2}, \beta b_{1}, \beta b_{2}, \cdots, \beta b_{t}, \mu a_{t+1}, \cdots, \mu a_{r}\right]^{T}(r>t)} \\
{\left[\mu a_{1}, \mu a_{2}, \beta b_{1}, \beta b_{2}, \cdots, \beta b_{r-1}, \beta b_{r}, \cdots, \beta b_{t}\right]^{T}(r<t)}
\end{array}\right.
$$

Then normalize ${ }^{C}$ as follows:

$$
c_{i}^{*}=\frac{C_{i}-C_{\min }}{c_{\text {max }}-c_{\text {min }}} \quad i=1,2, \cdots, r+t
$$

\section{SiMULATION AND VERIFICATION}

In this paper, we choose the Sallen-Key bandpass filter circuit which is the international standard test circuit to make the simulation[7]. The following figure shows the circuit diagram of Mutisium simulation and the algorithm is implemented by Matlab R2013a.

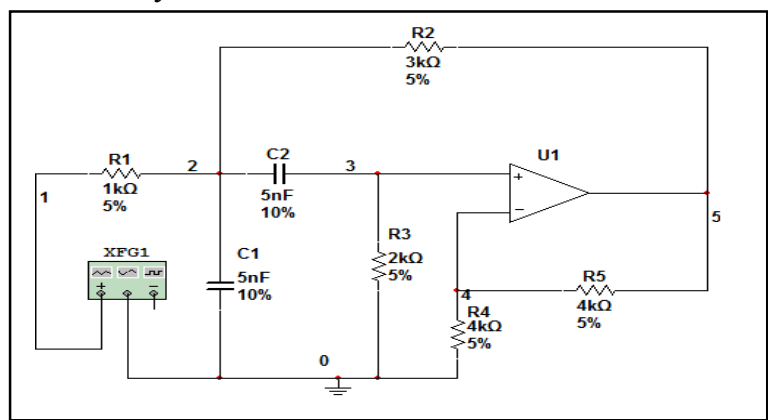

FIGURE II. SALLEN-KEY BANDPASS FILTER CIRCUIT DIAGRAM MODEL

The nominal values and tolerances for the components are shown in the figure. Node 1 is the input excitation point and node 5 is the only output response point. In the experiment, the excitation signal is selected as the square wave signal with base frequency of $10 \mathrm{KHZ}$, amplitude of $5 \mathrm{Vand}$ duty cycle of $10 \%$.

(1) The next figure is the result of the sensitivity analysis of each component to the output response point 5 in the Fig II.Through the sensitivity analysis, it can be seen that R2, R3, $\mathrm{C} 1, \mathrm{C} 2$ have high sensitivity. So select the fault set includes R2 $\uparrow, \mathrm{R} 2 \downarrow, \mathrm{R} 3 \uparrow, \mathrm{R} 3 \downarrow, \mathrm{C} 1 \uparrow, \mathrm{C} 1 \downarrow, \mathrm{C} 2 \uparrow, \mathrm{C} 2 \downarrow$ and normal mode a total of 9 kinds of failure modes. In this article, the deviation is set to $50 \%$.

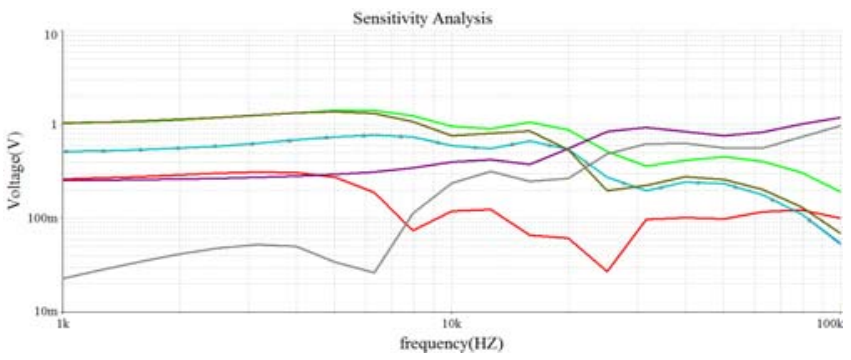

FIGURE III. SENSITIVITY ANALYSIS CHART

(2) Obtain original data and preprocess it. Under the Multisim10.0 simulation environment, the Monte Carlo analysis was carried out 30 times for each fault mode to get the original sampling data. 15 of these were for training and 15 were for testing. The next figure is 30 Monte Carlo simulation analysis of $\mathrm{R} 2 \downarrow$ and $\mathrm{C} 1 \uparrow$. The component parameters are subject to Gaussian distribution.

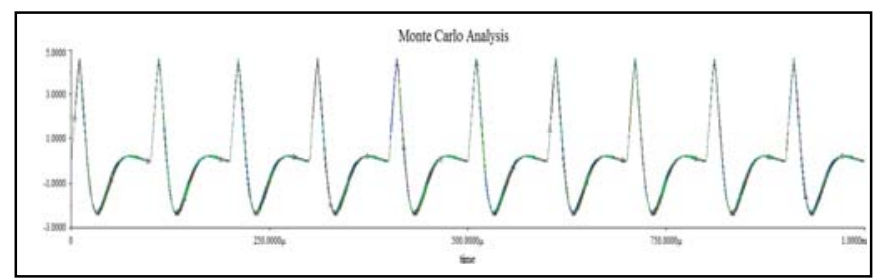

FIGURE IV. 30 MONTE CARLO SIMULATION RESULTS.

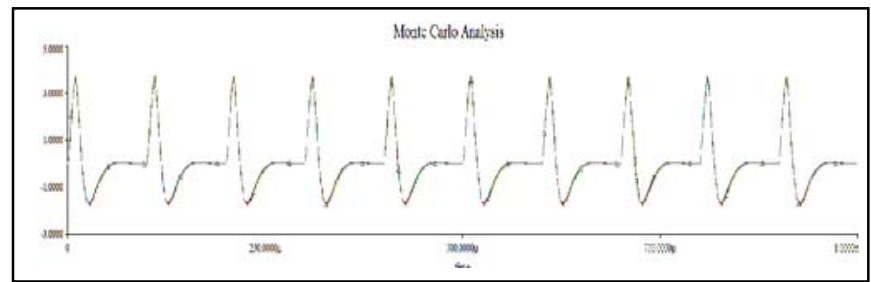

FIGURE V. 30 MONTE CARLO SIMULATION RESULTS

According to the sampling theorem, a total of 200 points were collected to obtain a scale of $270 \times 200$ raw data. And then wo do the difference with the standard signal to get the difference signal.

Make feature extraction of the signal.

1. Do PCA feature extraction. It is found that when the number of principal elements is 8 , the cumulative principal component contribution rate has reached more than $90 \%$, so the principal component analysis of the extracted feature vector sample size can be 8 .

2. Make wavelet packet energy extraction of the difference signal. Firstly, the wavelet packet is selected according to formula (7). In this paper, we choose Haar, $\mathrm{db} 2$ and $\mathrm{db} 3$ wavelet basis functions to calculate the characteristic deviation $D_{b}$. The results are shown in Table I. 
TABLE I. DIFFERENT WAVELET BASE FEATURE DEVIATION

\begin{tabular}{|c|c|c|c|c|}
\hline $\begin{array}{c}\text { Basis } \\
\text { function }\end{array}$ & $\mathrm{R} 2 \uparrow$ & $\mathrm{R} 2 \downarrow$ & $\mathrm{R} 3 \uparrow$ & $\mathrm{R} 3 \downarrow$ \\
\hline $\mathrm{Haar}$ & 0.0659 & 0.4759 & 19.6051 & 5.2957 \\
\hline $\mathrm{db} 2$ & 0.0951 & 0.6897 & 28.3846 & 7.6689 \\
\hline $\mathrm{db} 3$ & 0.1097 & 0.7977 & 32.8016 & 8.8667 \\
\hline $\begin{array}{c}\text { Basis } \\
\text { function }\end{array}$ & $\mathrm{C} 1 \uparrow$ & $\mathrm{C} 1 \downarrow$ & $\mathrm{C} 2 \uparrow$ & $\mathrm{C} 2 \downarrow$ \\
\hline Haar & 0.3305 & 4.4593 & 0.0002 & 0.022 \\
\hline $\mathrm{db} 2$ & 0.4777 & 6.4567 & 0.0003 & 0.0314 \\
\hline $\mathrm{db} 3$ & 0.5521 & 7.4665 & 0.0004 & 0.0365 \\
\hline
\end{tabular}

As can be seen from the table, the feature deviation of $\mathrm{db} 3$ wavelet base is greater than the first two. So, Using the db3 wavelet basis function in the wavelet toolbox of Matlab to make 3-layer wavelet packet energy feature extraction of preprocessed signal, The energy in 8 frequency bands are calculated and normalized, and the 8 dimensional feature vectors are obtained. The following figure shows the wavelet packet decomposition tree structure. Table II is the normalized energy feature values of the wavelet packet extraction of some fault models.

The next is the original signal and reconstructed signal comparison chart of one of the 30 Monte Carlo simulation results of the R2 $\downarrow$ model.

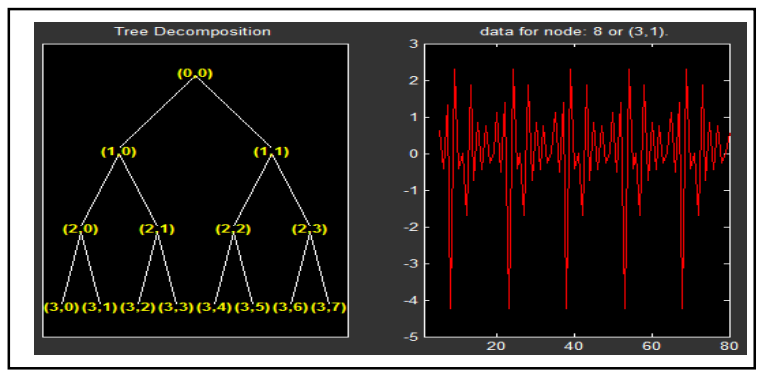

FIGURE VI. LEVEL WAVELET PACKET DECOMPOSITION TREE STRUCTURE

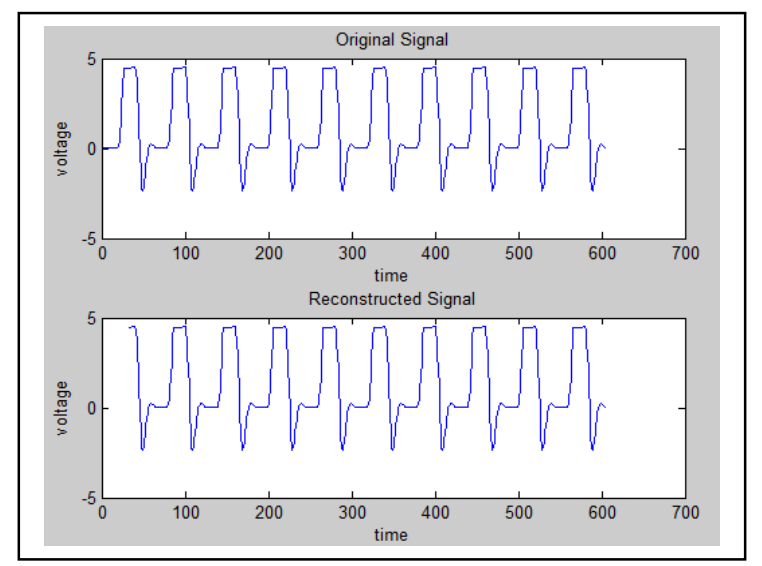

FIGURE VII. ORIGINAL SIGNAL AND RECONSTRUCTED SIGNAL COMPARISON CHART
TABLE II. NORMALIZED WAVELET PACKET ENERGY EIGENVALUES

\begin{tabular}{|c|c|c|c|c|}
\hline & $\mathrm{H}(3,0)$ & $\mathrm{H}(3,1)$ & $\mathrm{H}(3,2)$ & $\mathrm{H}(3,3)$ \\
\hline$R 2 \uparrow$ & 0.2001 & 0.9787 & 0.0340 & 0.0277 \\
\hline$C 1 \downarrow$ & 0.2211 & 0.9747 & 0.0032 & 0.0056 \\
\hline & $\mathrm{H}(3,4)$ & $\mathrm{H}(3,5)$ & $\mathrm{H}(3,6)$ & $\mathrm{H}(3,7)$ \\
\hline$R 2 \uparrow$ & 0.0013 & 0.0030 & 0.0034 & 0.0016 \\
\hline$C 1 \downarrow$ & 0.0010 & 0.0001 & 0.0001 & 0.0002 \\
\hline
\end{tabular}

(3) The extracted feature vectors are fused according to the three models proposed in this paper. And then, they are fed into support vector machine classifiers for fault diagnosis. First, we need to use SVM-Train to train the SVM model. Under the Support Vector Machine (SVM) toolbox of Matlab, we can get the structure of SVM by training fault feature samples. In this paper, the RBF kernel function is chosen. The parameters $\mathrm{C}$ and $\gamma$ are optimized by Grid.py tool[8]. After comparison, we choose $\mathrm{C}=8$ and $\gamma=1024$. Then, the test sample is input into the trained SVM, and the SVM-Predict function is called to classify the diagnosis.

(4) Analysis of simulation results

The following table shows the diagnostic accuracy of the different fusion model factors:

TABLE III. DIAGNOSTIC ACCURACY OF DIFFERENT FUSION FACTORS

\begin{tabular}{|c|c|c|c|c|c|c|}
\hline$\mu$ & 0.4 & 0.43 & 0.45 & 0.47 & 0.49 & 0.6 \\
\hline $\mathrm{W}$ & 83.36 & 86.58 & 88.15 & 88.09 & 85.97 & 82.14 \\
\hline $\mathrm{O}$ & 89.30 & 90.47 & 90.68 & 90.53 & 89.24 & 85.33 \\
\hline $\mathrm{T}$ & 85.28 & 88.23 & 89.11 & 88.98 & 87.16 & 84.13 \\
\hline
\end{tabular}

The larger the fusion factor $\mu$ is, the more the PCA feature is in the fused feature vector. when the $\mu$ is 0.46 or so, the diagnostic accuracy rate of each model reached the highest. After comparison between the three models, one-one cross model diagnosis the highest correct rate. The next table is the average diagnostic results of 50 different random experiments for each feature extraction method when the $\|=0.46$.

TABLE IV. DIAGNOSTIC RESULTS OF DIFFERENT FEATURE EXTRACTION METHODS

\begin{tabular}{|c|c|c|c|}
\hline Method & PCA & wavelet packet & fusion \\
\hline Correct rate (\%) & 85.14 & 89.19 & 95.74 \\
\hline Error rate (\%) & 14.86 & 10.81 & 4.26 \\
\hline Time (s) & 0.27 & 0.26 & 0.46 \\
\hline
\end{tabular}

It can be seen that although the diagnosis time is longer, the diagnostic accuracy of the fusion model is significantly higher than the diagnostic accuracy when using alone. This method is very useful for analog circuit fault diagnosis which the realtime requirements are not very high.

\section{CONCLUSION}

According to the idea of information fusion, this paper presents the method of fault feature extraction based on principal component analysis and wavelet packet analysis, and constructs three different feature vector fusion models. Through the support vector machine training and parameter 
optimization selection, the fusion feature vectors are then fed into support vector machine classifier for simulation and verification. The results show that the method can effectively improve the accuracy of fault diagnosis.

\section{REFERENCES}

[1] J. Y. Tang, Study on Feature Extraction and Support Vector Machines Ensemble Method in Fault Diagnosis for Analog Circuit[D].ChengDu, University of Electronic Science and Technology of China,2009.

[2] AMINAN M, AMINAN F. Neural network based analog-circuit fault diagnosis using wavelet transform as preprocessor [J].IEEE Trans on Circuits Syst-II, 2000, 44(3):151-156.

[3] T. Xie, The Research on Fault Diagnosis for Analog Circuits Based on(Multi) Wavelet(Packet) Analysis, Neural Networks and Optimization[D].ChangSha, Hunan University,2011.

[4] Y. K. Sun, Study on Fault Diagnosis in Analog Circuits Based on Support Vector Machine[D], ChengDu, University of Electronic Science and Technology of China,2009.

[5] X. He, H. L. Wang, J. H. Lu, Analog circuit fault diagnosis method based on preferred wavelet packet and ELM[J]. Chinese Journal of Scientific Instrument,2013,34(11):14-19.

[6] M. Y. Wu, Research on Analog Circuit Fault Diagnosis Based on Information Fusion[D].DaLian, Dalian University of Technology,2011.

[7] M. Li, S. W. Zhou, Simulation and Analysis for Analog Circuits Fault Diagnosis Using PSpice and Matlab[J]. Journal of Electrical \& Electronic Education, 2010,32(6): 60-63

[8] Y. F. Xiao, Z. R, Liu. M.Li, Analog circuit fault diagnosis based on wavelet packet energy entropy and SVM[J]. Electronic Measurement Technology,2011,34(6):110-113 